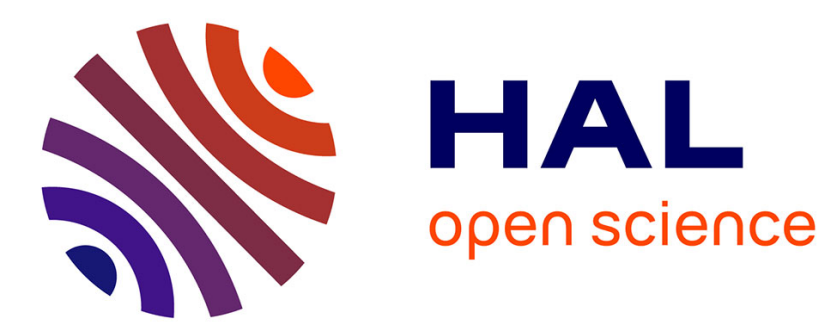

\title{
Signification géographique des implantations industrielles décentralisées en province
}

Thérèse Saint-Julien

\section{To cite this version:}

Thérèse Saint-Julien. Signification géographique des implantations industrielles décentralisées en province. Annales de géographie, 1973, 82 (453), pp.557-575. 10.3406/geo.1973.18912 . hal-02546680

\section{HAL Id: hal-02546680 \\ https://hal.science/hal-02546680}

Submitted on 18 Apr 2020

HAL is a multi-disciplinary open access archive for the deposit and dissemination of scientific research documents, whether they are published or not. The documents may come from teaching and research institutions in France or abroad, or from public or private research centers.
L'archive ouverte pluridisciplinaire HAL, est destinée au dépôt et à la diffusion de documents scientifiques de niveau recherche, publiés ou non, émanant des établissements d'enseignement et de recherche français ou étrangers, des laboratoires publics ou privés. 


\section{Signification géographique des implantations industrielles} décentralisées en province

Thérèse Saint-Julien

\section{Citer ce document / Cite this document :}

Saint-Julien Thérèse. Signification géographique des implantations industrielles décentralisées en province. In: Annales de Géographie, t. 82, n453, 1973. pp. 557-575;

doi : https://doi.org/10.3406/geo.1973.18912

https://www.persee.fr/doc/geo_0003-4010_1973_num_82_453_18912

Fichier pdf généré le 30/03/2018 


\title{
Résumé
}

La politique de décentralisation industrielle semble avoir joué depuis une vingtaine d'années en France un rôle important dans la mobilité géographique de certaines activités industrielles. Les pouvoirs publics ont encouragé un processus, entrevu autant comme une émigration des activités industrielles localisées en région parisienne que comme une orientation hors de cette dernière des effets de leur croissance. Ces acceptions sont d'autant plus vagues que l'on s'attache à l'étude de la projection spatiale de ce mouvement. Les géographes français, très tôt intéressés par ce dernier, se sont jusqu'ici peu préoccupés de l'analyse des formes d'implantations et de leur dynamisme de croissance. II nous est apparu nécessaire de présenter les aspects les plus caractéristiques de la complexité géographique des opérations de transferts industriels observés afin de mieux dégager les formes originales des implantations décentralisées en province, lesquelles doivent être appréhendées dans la dynamique même de leur évolution.

\begin{abstract}
The geographic significance of the decentralized industrial settlements in the provinces.

The policy of industrial decentralization seems to have played an important role in the geographical mobility of some industrial activities in France for twenty years or so. The administration supports a system which is considered as a move of industrial activities located in the Paris region, as much as an orientation far from this region of the effects of their growth. Those definitions are so much vague as the study of the spatial projection of this move is looked upon. The French geographers were very soon interested in that move and did not worry very much about the analysis of the settlements forms and of their growth dynamism. It appeared to us as a necessity to present the most characteristic aspects of the geographical complexity the industrial moves show in order to determine better the original types of decentralized settlements which must be studied through the very dynamic of their evolution.
\end{abstract}




\title{
Signification géographique des implantations industrielles décentralisées en province
}

\author{
par Thérèse Saint-Julien
}

Assistante à l'Université de Paris I

\begin{abstract}
La politique de décentralisation industrielle semble avoir joué, depuis Une vingtaine d'années en France, un rôle important dans la mobilité géograohique de certaines activités industrielles. Cette politique a suscité beaucoup 49. 'intérèt, d'espoirs, de critiques, de bilans, de publications diverses. On monnaît pourtant encore assez mal la forme, les structures, l'évolution de (e) que l'on peut en première approximation appeler l'implantation industrielle décentralisée.

Après avoir cherché quelle signification ont donné les pouvoirs publics a l'expression "décentralisation industrielle», et retenu les enseignements des études géographiques qui en ont examiné les traductions provinciales, nous nous sommes attachée à voir, à l'examen des faits, quelles réalités géographiques peut recouvrir cette formule.
\end{abstract}

\section{SIGNIFICATION DONNÉE EN FRANCE PAR LES POUVOIRS PUBLICS À L'EXPRESSION «DÉCENTRALISATION INDUS- TRIELLE »}

L'étude des formes et de l'évolution des implantations industrielles décentralisées pose une première question d'ordre terminologique : quel sens ont donné à l'expression "décentralisation industrielle " les auteurs de cette politique qui est en France, depuis vingt ans, à l'origine d'une migration des activités industrielles de la région parisienne vers la province? Cet examen préliminaire nous paraît être indispensable à toute approche des traductions spatiales de ce mouvement. Nous avons donc pour cela systématiquement analysé les textes législatifs, circulaires et déclarations officielles, qui ont 
réorganisé et favorisé en France depuis 1950, dans le cadre général de l'Aménagement du Territoire, une décentralisation des activités industrielles. Cette analyse nous a permis d'observer que le législateur éprouve peu la nécessité de définir l'objet même de l'appareil législatif qu'il met en place, puis modifie. Sa signification ne peut souvent être déduite que de l'analyse de la situation qui tend à en justifier la mise en œuvre et des objectifs qui lui sont assignés.

L’idée maîtresse qui, à l'origine, présida à cette politique française de la décentralisation industrielle est que celle-ci doit favoriser une implantation rationnelle des industries sur l'ensemble du territoire. Dès février 1950, E. Claudius-Petit dans un rapport du ministère de la Reconstruction et de l'Urbanisme au Conseil des ministres ${ }^{1}$, analysant quelques-uns des désordres dont souffre la France, affirme : "désordre... qui sous quelque angle qu'on l'envisage a son origine dans une mauvaise répartition des activités sur le territoire ". Deux composantes essentielles de cette irrationalité retiennent particulièrement l'attention des pouvoirs publics : la première s'exprime par la congestion des grandes agglomérations qui à l'échelle nationale est essentiellement le fait de l'agglomération parisienne; la seconde, qui lui est liée, se traduisant par un lent et profond dépérissement des provinces françaises. De ces deux constats, découle dans une perspective d'aménagement. du territoire la nécessité de promouvoir une décentralisation industrielle.

"Il faut endiguer le courant qui porte toutes les forces vives du pays vers les grands centres, recréer des sources de vie dans les régions dont les ressources sont insuffisamment utilisées et qui tendent à devenir désertes ", écrit E. Claudius-Petit ${ }^{2}$ qui préconise en outre de favoriser la décentralisation industrielle.

Suite logique de cette déclaration, émane du mème ministère, en décembre 1950 , un premier rapport ${ }^{3}$ concernant le développement de l'industrie en France sur un plan décentralisé, qui, fail assez rare, aborde ouvertement la question terminologique. Il se réfère pour cela aux travaux de G. Dessus ${ }^{4}$. Celui-ci a dès 1943 défini la décentralisation, préconisée comme une industrialisation faite aux dépens des régions congestionnées. "Nous proposons d'employer le terme de congestion (ou agglomération) pour désigner la réunion en un même lieu d'un grand nombre d'hommes se consacrant à des activités diverses - encore faudrait-il distinguer la congestion proprement industrielle et la congestion urbaine générale pouvant résulter de la congestion d'activités autres qu'industrielles [...]. Fn réalité, c'est contre la congestion sous l'une quelconque de ses formes que l'on entend lutter

1. Pour un plan d'aménagement du territoire, Paris, Ministère de la Reconstruction et de l'Urbanisme, 1950.

2. Ibid.

3. Amenagement du teritoire: 1er rapport, Paris, Ministère de la Reconstruction et le l'Urbanisme, dèr. 1950.

4. Rapports et travaux sur la decongestion des centres industriels; fascicule no $1:$ Introductirn à l'étude de la localisation industrielle, par (;. Dessts, Dólégation générale de l'équipement national, 1943. 
lorsqu'on parle de décentralisation ", peut-on lire dans l'Introduction à l'étude des localisations industrielles. Son auteur a en outre distingué le desserrement, qui est un "éloignement à l'échelle régionale ", de la décentralisation, "éloignement à l'échelle nationale " et qui consiste à "industrialiser aux dépens des régions congestionnées un certain nombre de petites villes choisies pour les commodités géographiques qu'elles présenteraient ". Le rapport du ministère de la Reconstruction tient pour acquise dans son ensemble cette terminologie, repousse toute solution de desserrement pour remédier à la congestion de l'agglomération parisienne, rappelle que la décentralisation peut autant s'appliquer à des entreprises à créer qu'à des entreprises à déplacer et procède à l'examen des régions à industrialiser et de la dimension des apports industriels souhaitables.

L'examen de ces rapports et études préliminaires à la mise en œuvre de cette politique nous a paru ici nécessaire car ils ont profondément influencé les orientations et la signification qu'à leur suite celle-ci prendra pour de nombreuses années.

Il faut attendre la fin de l'année 195 \& et surtout 1955 pour qu'un vaste ensemble de mesures soit pris qui rend effective la politique annoncée. I.a notion de décentralisation industrielle y est explicitement introduite de la même manière. L'exposé des motifs du décret 1 de janvier 1955, "tendant à une meilleure répartition des industries sur l'ensemble du territoire " par l'institution d'une demande d'agrément pour toute création ou extension industrielle en région parisienne, développe largement les thèmes précédemment évoqués. Y sont rappelés, tant les graves inconvénients suscités par la congestion industrielle de l'agglomération parisienne (immigration, insuffisance des logements et des services, coût et faible qualité de cette croissance mal dominée), que l'accélération des crises et désertions d'une grande partie $\mathrm{du}$ territoire national. Le texte devient alors très clair et fait pour quelques années de la décentralisation industrielle la pièce maîtresse de l'aménagement du territoire en France. On peut y lire en effet : " le seul moyen qui s'offre aux pouvoirs publics pour lutter à la fois contre la désertion de certaines régions et contre la surpopulation des grandes agglomérations consiste à diriger vers les premières une fraction des créations ou des extensions d'entreprises qui se localisent actuellement dans les secondes, dans une proportion très supérieure à celle que ces dernières tiennent dans l'activité industrielle nationale ".

La décentralisation industrielle semble donc être conçue comme une tentative de transfert géographique de la croissance industrielle de la région parisienne, très précisément délimitée par ce décret, vers les zones les plus défavorisées, mais sans que soit ici reprise la distinction entre éloignement à l'échelle régionale et éloignement à l'échelle nationale. Le législateur paraît peu se préoccuper des caractères de la croissance dont il préconise et encourage le transfert, tant à l'échelle de l'entreprise industrielle elle-même que

1. Décret no 55-36 du 5 janv. $1955 ;$ J.O. du 8 janv. 1955. 
de l'industrie française dans son ensemble; il rappelle simplement à plusieurs reprises dans les textes des décrets du 30 juin $1955^{1}$, "tendant à stimuler la mise en valeur des régions souffrant de sous-emploi ou d'un développement économique insuffisant ", que dans le cadre de la décentralisation industrielle peuvent ètre réalisées par les entreprises des opérations de transferts, de créations ou d'extensions. Il serait toutefois peut-être souhaitable d'accorder au décrel $^{2}$ visant à favoriser la décentralisation des établissements scientifiques et techniques une place à part, puisqu'il tend à encourager le transfert hors de la région parisienne d'établissements dont la nouvelle localisation peut avoir une influence très positive sur l'importance de l'émigration industrielle souhaitée. Faut-il voir là un indice d'une approche plus qualitative des transferts préconisés?

Cette absence de précision terminologique est profondément dépendante, nous semble-t-il, de la nature des moyens mis en œuvre pour apporter par la décentralisation industrielle des solutions aux déséquilibres dénoncés. Pour freiner la croissance de l'agglomération parisienne des mesures sont prises qui visent à $\mathbf{y}$ contrôler toute extension des surfaces industrielles et à en encourager la réduction : demande d'agrément pour toute création ou extension $^{3}$, institution de sanctions aux infractions, de redevances pour tout accroissement ${ }^{5}$ et de primes pour toutes diminutions ${ }^{6}$. Parallèlement, les pouvoirs publics s'attachent à favoriser l'industrialisation régionale dans un souci clairement exprimé de création d'emplois, qui reste pour les législateurs un facteur essentiel de revivification des provinces françaises. Dans cette optique ont été publiés en 1954 et en $1955^{7}$ les très importants décrets qui ont donné à la politique de décentralisation industrielle sa deuxième dimension; mais ces mesures ne lui sont pas spécifiques, même si le législateur suppose dans ce domaine son rôle prépondérant. Dans le premier champ d'action des pouvoirs publics, la décentralisation est associée à une diminution des surfaces industrielles; dans le second, elle est le plus souvent perçue par la croissance des emplois industriels. La décentralisation industrielle dans son ensemble, dans son existence du point de départ au point

1. Décrets du 30 juin $1955 ; J . O$. du 2 juillet 1955 ; décret $n^{\circ} 55-873$ relatif à l'établissement de programmes d'action régionale :

décret no $55-87 /$ relatif à la garantie de l'Etat et aux bonifications d'intérêt en matiore de conversion de l'industrie, de décentralisation industrielle, de développement régional :

décret no 55-875 relatif à la création d'un Fond de développement icononique et social ; décret no 55-876 relatif à la création des S.D.R. ;

décret no 55-878 relatif à la création de la prime spéciale d'équipement;

décret no 55-879 relatif aux allégements fiscaux en faveur de l'expansion économique régionale ; décret no 55-883 facilitant la décentralisation des activités administratives, scientifiques ct techniques.

2. Décrets du 30 juin 1955 "tendant a stimuler la mise en valeur des régions souffrant de sous-emploi ou d'un développement économique insuffisant".

3. Décret no $55-36$ du 5 janv. 1955;

decret no $55-883$ du 30 juin $1955 ;$

décret $n^{\circ} 58-1460$ du 31 déc. 1958

décret no 58-1461 du 31 déc. 1958.

4. Décret no 58-1446 du 31 déc. 1958

5. Décret no $60-9.41$ du 5sept. 1960 .

i. Id.

j. I écrets du 30 juin $195 \%$. 
d'arrivée, n'est jamais définie et appréhendée en tant que telle par les mesures qu'elle inspire pourtant.

Clairement annoncées au Parlement dès l'automne 1963 par O. Guichard1 au cours d'un important débat parlementaire, de profondes modifications ont été apportées en 196/ au système des aides à la décentralisation et à l'industrialisation régionale. Ces nouvelles mesures, qui s'inscrivent dans un ensemble marquant bien un tournant dans la politique d'aménagement du territoire en France, sont restées en vigueur pour l'essentiel jusqu'en 1971, et les récentes orientations prises n'en remettent pas en cause les directions profondes $^{2}$. Ces modifications n'apportent cependant pas à la notion de décentralisation industrielle un éclairage nouveau. Parallèlement au maintien des mesures restrictives tendant à freiner l'expansion industrielle en région parisienne, l'ensemble des incitations favorisant l'industrialisation régionale est réorganisé en fonction de pôles et de zones choisis et connus de tous sur lesquels elles sont concentrées, et ce, bien entendu, quelle que soit l'origine géographique de l'entreprise qui s'implante ou se développe. "I'action menée au moyen de la prime de développement industriel et de la prime d'adaptation industrielle devra continuer à s'insérer dans un dispositif d'ensemble comportant des aides spécifiques aux décentralisations de la région parisienne ", est-il noté dans le rapport au Premier ministre ${ }^{3}$, introduisant un des décrets les plus importants. Parallèlement est instituée une aide propre à la décentralisation qui tend à dédommager les entreprises d'une partie de leurs frais de déménagement de matériel industriel de la région parisienne vers la localisation nouvelle 4 . Cette indemnité spécifique de certaines décentralisations, s'accompagnant d'un transfert de matériel industriel et d'une libération au lieu d'origine d'une surface industrielle supérieure ou égale à $500 \mathrm{~m}^{2}$ (des aménagements ont par la suite été apportés à cette dernière condition), ne donne pourtant à cette notion aucune signification nouvelle. Faut-il, toutefois, voir dans les limites imposées à toute localisation provinciale choisie par l'industriel qui veut en bénéficier quelque rappel que la décentralisation ne peut être un simple éloignement à l'échelle régionale ou n'est-ce là que volonté de ne point encourager, par des incitations financières, un mouvement qui s'est très largement développé à l'intérieur même du Bassin Parisien et dont certains regrettent l'ampleur?

Une grande imprécision terminologique entoure donc encore aujourd'hui ce phénomène que les pouvoirs publics n'ont cessé d'encourager depuis vingt ans, sans s'attacher à en dégager une signification précise. La décentralisation semble avoir été entrevue par eux, autant comme une émigration

1. Un Grand Débat parlementaire, 23 nov. 1963, Paris, La Documentation française, 1963.

2. Décret no $72-270$ du 11 avril 1972 relatif à la prime de développement régional.

3. Décret no 64-440 du 21 mai 1964 instituant une prime de développement industriel et une prime d'adaptation industrielle. (Ce décret a fait l'objet de réaménagements ultérieurs : décrets no 65-99, 65-329, 65-849, 65-1176,66-289, 67-939,67-941,67-942,67-943, 69-285.)

4. Décret no 6\$-441 du 21 mai 1964 instituant une indemnité de décentralisation (ce décret $\rightarrow$ fait l'objet de modifications ultérieures : décrets no 65-585, 66-290).

ANN. DE GEOG. LXXXII' ANNEE. 
des activités industrielles de la région parisienne, que comme une orientation hors de cette dernière des effets de leur croissance, mais cette dernière acception demeure très vague.

\section{LES GÉOGRAPHES ET L'APPROCHE DE L'IMPLANTATION INDUSTRIELLE DÉCENTRALISÉE EN PROVINCE}

Les géographes français se sont très tôt penchés sur la traduction géographique provinciale de cette politique, ou plus généralement de ce mouvement. Innombrables sont les publications qui en font mention : il est bien peu d'ouvrages, d'articles de géographie régionale urbaine ou industrielle de la France qui n'y fassent référence depuis une quinzaine d'années. Il nous a paru souhaitable, dans un premier temps de notre démarche, de noter les enseignements de ces études. Toutefois, seules celles dont l'objet premier est la décentralisation industrielle, ou celles qui y ont consacré de larges développements susceptibles d'enrichir notre approche, ont retenu notre attention.

\section{A. Des bases d'analyses très diverses}

Les géographes abordant l'examen des formes d'industrialisation régionale par décentralisation en France se sont souvent peu préoccupés de questions terminologiques; mais, en l'absence d'un consensus général sur la signification de l'expression décentralisation industrielle, leurs bases d'analyse sont très diverses et plus ou moins sensibles selon les cas à l'un des aspects.

Certains n'ont pris en considération que les implantations issues d'un transfert effectif d'activités industrielles de la région parisienne vers la ou les localisations provinciales. F. J. Gay écrit dans le commentaire de la carte des créations industrielles en Normandie de 195́́ à 1964 (1) ${ }^{1}$ : "La décentralisation industrielle concerne les activités qui ont quitté la région parisienne pour s'établir en province, généralement avec le concours de l'État ", et M. M. Krust (18) analysant à Reims les traductions de ce phénomène note : "Nous n'étudierons que les transferts d'activités industrielles de la région parisienne à Reims, et non ceux des usines antérieurement installées déjà en province, même lorsque le siège social de l'entreprise est situé à Paris ". Ces deux auteurs ne semblent pas retenir l'acception des pouvoirs publics selon laquelle il peut $\mathrm{y}$ avoir décentralisation par création nouvelle et par extension.

A. Frémont, M. Ambois et M. Chesnais remarquent à l'observation des faits à Argentan (14) la diversité des significations possibles (transferts, absence de transferts...), mais justifient l'emploi général de l'expression en

1. Les chiffres entre parenthèses renvoient à la bibliographie placée en fin d'article. 
précisant que dans tous les cas observés, à l'origine de l'implantation provinciale, réside une gène pour la survie ou l'extension en région parisienne, et qu'un lien original est conservé avec le milieu d'origine, à savoir le maintien a Paris des organes de direction, de commercialisation et de recherche. La décentralisation industrielle est ici définie par les motivations du transfert, le milieu géographique d'origine et la nature des relations conservées avec celui-ci.

D'autres ont pris cette expression dans un sens très large. P. George, tout en dénonçant l'ambiguïté, précise en 1961 (15) "qu'il ne s'agit pas tant de déplacer des activités existantes et prospères que d'écarter de nou. velles implantations, et de substituer à des processus d'accroissement sur place des processus d'éclatement géographique "; en 1967 (6) il estime que l'expression est appliquée " à toute implantation hors de la région parisienne d'un établissement dont le siège social ou l'agence principale est à Paris". G. Burgel (12) semble aller plus loin encore : pour lui en effet "la décentralisation recouvre deux réalités : le transfert hors de la région parisienne d'établissements qui s'y trouvaient installés (stricto sensu) et toute création industrielle réalisée hors de la région parisienne (lato sensu)". Il ajoute : " mais c'est un même fait géographique qu'il convient d'étudier dans son ensemble : l'industrie décentralisée ". Dans ce deuxième cas l'acception devient très vague, et la référence à la région parisienne a même changé de nature. Il ne s'agit plus nécessairement d'une industrialisation faite aux dépens de la région parisienne ou en relation avec elle mais tout simplement réalisée hors de celle-ci.

P. Riquet (21) voit dans une signification trop large de l'expression " un abus de langage". Depuis fort longtemps, écrit-il, "des établissements de province sont commandés depuis Paris, sans que le mot décentralisation ait jamais été avancé. Des sociétés étrangères qui créaient un établissement industriel en France ont même obtenu par l'intermédiaire de leur bureau parisien le bénéfice des avantages réservés aux opérations de décentralisation ". Il s'attache donc à une définition plus restrictive, mais qu'il reconnaît arbitraire, à savoir "l'aptitude à l'implantation industrielle dont l'initiative revient à des entreprises parisiennes " qu'il tente de mesurer à l'aide des dossiers établis par le ministère de la Construction.

Il ressort de ce qui précède que la plupart des géographes ont bien perçu la décentralisation industrielle comme un moyen d'industrialiser les régions françaises aux dépens de la croissance industrielle parisienne. Mais, compte tenu de l'imprécision donnée par les pouvoirs publics à cette notion de croissance transférée, ils l'ont interprétée de manière plus ou moins restrictive, et leur analyse de la répartition et des formes des implantations décentralisées s'en trouve parfois sensiblement influencée. 


\section{B. L’implantation décentralisée perçue par sa localisation et ses emplois créés}

I a traduction provinciale de la décentralisation industrielle a été avant tout perçue dans les études géographiques sous les angles de la localisation et de la création d'emplois, ce qui ne saurait surprendre si l'on se réfère aux objectifs assignés par les pouvoirs publics à cette politique depuis vingt ans.

L'examen des localisations concernées par cette migration originale des activités industrielles a donc de leur part fait l'objet d'une approche attentive, mais à des échelles et selon des orientations différentes.

Quelques auteurs (5) (7) (8) (15) se sont intéressés pour l'ensemble du territoire à la répartition générale de ces industries nouvellement implantées; leur réflexion s'accompagne de cartes établies à partir de bilans départementaux (nombre d'opérations réalisées, nombre d'emplois créés), publiés soit par le ministère de la Construction soit par la Iélégation à l'Aménagement du Territoire. Mais à cette échelle le document le plus original est la carte présentée par l'Atlas de P'aris et de la région parisienne (2). Ses auteurs s'altachent à cartographier, en les localisant pour l'ensemble de la France, chacune des implantations décentralisées de 1954 à 1963, les définissant par leur taille (nombre d'emplois créés), leur branche d'activité et le type de transfert dont elles sont issues.

Les études régionales de localisation sont plus nombreuses et très disparates. Y. Babonaux (3), à propos de l'analyse des localisations industrielles dans les villes et régions de la Loire moyenne, dresse un bilan " des apports extérieurs dont l'essentiel est lié à la décentralisation ". Il cartographie pour la région étudiée l'importance du phénomène. A la mème époque les auteurs du cahier de l'I.A.U.R.P. (21), précédemment cité, répertorient dans un rayon de 250 kilomètres autour de Paris les localisations concernées et établissent des rapports entre l'importance des transferts et la taille des agglomérations choisies. Ie son côté F. J. Gay précise à propos du commentaire de la carte des créations industrielles en Normandie de 1954 à 1964: "Pour l'essenticl, l'évolution des localisations ainsi exprimée s'explique par ce que l'on appelle la décentralisation industrielle ". Nous disposons de quelques travaux où la répartition et les localisations ont été abordées à l'échelle d'un département (23) (2/4) (26). Beancoup moins systématiques, "Notes sur la décentralisation industrielle entre Seine et l.oire " (17), "Bilan de la décentralisation industrielle en Eure-et-1 coir" (13), "I a main-d'ouvre des établissements décentralisés: l'exemple de l'Eure" (12), témoignent d'un même souci de localiser les centres atteints, et Cl. Parry (20) dresse une carte des usines créées par la Radiotechnique à l'ouest de Paris de 1953 a 1957. Enfin, sont à mentionner quelques études précisément conduites en fonction d'une localisation déterminée : Argentan (14), Reims (18), I)ijon (16), Isa Rochelle (22). 
Cette approche géographique des localisations concernées par la décentralisation industrielle suggère quelques remarques : M. Rochefort (8) parle de "décentralisation boulevard ", P. George (15) constate : "les représentations carlographiques que l'on peut donner des implantations industrielles nouvelles dirigées de Paris suggèrent plutôt l'idée d'un desserrement du centre parisien que celle d'une décentralisation au sens plein du terme ", et l'on peut lire dans la conclusion de l'étude de l'I.A.U.R.P. (21) : "La décentralisation apparaît comme la projection des structures géographiques de l'activité parisienne dans un espace plus vaste, et de préférence, pour le sud et l'ouest de la périphérie, comme un prolongement des tendances préférentielles du desserrement ". Nous retrouvons là, semble-t-il, comme un écho des distinctions entre décentralisation et desserrement étudiées par G. Dessus, reprises par le ministère de la Reconstruction en 1950, mais qui ne réapparaissent explicitement dans aucun texte législatif ultérieur. A vrai dire, cette référence à la seule distance géographique est-elle suffisante pour distinguer ces deux notions ? Ne s'agit-il pas moins de distances géographiques au sens strict que des rapports de ces dernières a vec les caractères économiques et géographiques de l'entreprise industrielle considérée et la croissance de l'agglomération parisienne elle-mème ?

Enfin rares sont les correspondances établies entre les localisations étudiées el l'évolution de la forme et de l'importance des aides accordées par l'État. Ses interventions et sa politique suggèrent quelques remarques parfois essentielles à l'échelle de la France entière (6) (7) (8) (9) (15) ; au niveau local la question n'est explicitement abordée qu'à propos des agglomérations de IJijon (16) et de La Rochelle (22).

I'analyse des emplois industriels a relenu l'attention de tous et a parfois été le seul critère choisi pour mesurer l'importance des implantations décentralisées, et aussi d'une certaine manière pour apprécier l'efficacité de cette politique (10) (12). A grande échelle quelques auteurs ont tenté d'en analyser les structures qualitatives (12) (14) (20) (22); à petite échelle seul le total des emplois créés par établissement est indiqué (1) (2) (20), à moins que la réflexion ne s'effectue à partir de bilans départementaux (5) (7) (8) (15) où disparait alors totalement la dimension de l'implantation décentralisée elle-mème.

Localisation et emplois créés ont bien été les critères de connaissance des implantations décentralisées les plus attentivement étudiées.

\section{Attributions, forme et évolution des implantations industrielles décentralisées en province}

D'autres aspects restent jusqu'ici beaucoup moins connus, beaucoup moins systématiquement explorés : il s'agit des attributions, de la forme de l'implantation décentralisée et de son évolution.

I es types de transfert ont suscité divers examens qui tendent à cerner la nature de cette mobilité Paris-province. Quelques études seulement se 
sont penchées sur la nature concrète des transferts el du passage Parisprovince qui en a résulté (14) (15) (20) (22). Toutefois leur analyse trop ponctuelle permet mal de cerner la diversité du phénomène. On doit à J. Bastic et I'. 'Trolliet d'une part (2), et à P. Riquet d'autre part (21), d'avoir tenté l'élablissement de typologies qui cherchent a caractériser le poids des activites transférées hors de la région parisienne par rapport à l'activité générale de l'entreprise. Plus ou moins complexes, marquées par l'optique générale des travaux dans lesquels elles s'insèrent, ces typologies présentent une réelle convergence, et leurs classifications ont été par la suite très souvent réutilisées; peut-ètre ne nous permettent-elles pas de saisir assez concrètement la nature du partage sur laquelle reposent les implantations provinciales.

Les traductions locales de la décentralisation restent tout aussi imprécises. Nous ne nous attarderons pas sur l'emploi très fréquent de l'expression opération, tant pour désigner l'action de transférer que le programme d'investissement provincial considéré, quand il ne s'agit pas tout simplement d'une tranche de ce programme, et ce indifféremment et sans précision. Gutre qu'il ne semble pas superflu de s'interroger sur la correspondance dans le temps et dans l'espace des deux types d'opérations, il nous faut bien constater que cette expression traduit peu le concret géographique, et remarquer que son utilisation très fréquente n'est pas étrangère à l'emploi qu'en ont fait les statistiques officielles de la décentralisation industrielle tant qu'elles ont été publiées, sans qu'en ait été très clairement défini le sens.

Pas davantage ne nous éclairent les expressions d'" entreprises décentralisées " ou d'“établissement décontralisé ». I a première (13) (1/4) ne peut manquer de faire naitre quelque confusion, assimilant une notion juridique et financière à une réalité spatiale dont la base peut ètre très parcellaire dans l'entreprise; la seconde ne cerne mieux qu'en apparence la réalité provinciale de la décentralisation.

Plus significatives des formes de cette dernière peuvent ètre les expressions qui, sous des vocables variés, semblent signifier que la décentralisation se traduit en province par la création de nouveaux établissements industriels : " installation de nouvelles industries (13), "implantation d'industries nouvelles " (17), "nouvelles implantations" (12), "industries nouvelles dirigées de Paris " (15), "création d'usines nouvelles " (12) (20), "création de nouveaux établissements"(21), etc. Peut-on en conclure que tous les transferts d'activites industrielles de la région parisienne vers la province se traduisent par la création de nouveaux établissements, ou s'agit-il là seulement de leur traduction la plus spectaculaire, la plus fréquente ? En outre, la part relative de cette dernière n'a-t-elle pas varié a vec le temps ? L'étude de M. M. Krust (18), quelques remarques faites par M. Chesnais (13) et Y. Babonaux (10) semblent justifier l'intérot de ces questions, mais ne permettent pas d'y apporter des réponses claires. Il ne faut de plus pas perdre de vue ici que cet examen reste essentiellement dépendant de la signification qu'ont donnée les diffirents auteurs à la décentralisation. 
Enfin la vie de ces établissements, la dynamique de cette forme originale d'industrialisation des régions françaises ne sont abordées qu'incidemment; l'étude de l'I.A.U.R.P. (21) chiffre pour le Bassin Parisien les cas de suppression d'établissements dits décentralisés, qui ont cessé leur activité au cours de la période étudiée; nous ne retrouvons d'autre part que deux mentions au demeurant significatives : les auteurs de l'étude de la décentralisation à Argentan (14) remarquent que " la décentralisation de Moulinex, de Bagnolet à Alençon, se produisit avant la guerre de 1939-1945 mais que l'installation d'une usine à Argentan correspond à une extension régionale de la société, qui a partir du centre de fabrication d'Alençon essaime des satellites sur un axe nord-sud"; J. Gouhier note (17) à propos des sociétés Moulinex et Ferodo dont il décrit l'évolution : "ce sont deux exemples originaux d'évolution de la décentralisation avant la lettre ".

Par ce rapide examen bibliographique, nous avons tenté de dégager les seuls aspects de l'implantation industrielle décentralisée qui ont été jusqu'ici en France les plus attentivement examinés par les géographes; ce faisant nous avons pu remarquer quelques-uns des problèmes posés par ces études.

\section{RÉFLEXIONS A PROPOS DE LA SIGNIFICATION GÉOGRAPHIQUE DES FORMES, STRUCTURES ET ÉVOLUTION DES IMPLANTATIONS DECENTRALISÉES EN PROVINCE}

L'analyse de plusieurs centaines de cas d'entreprises parisiennes qui de 1963 à 1970 ont totalement ou partiellement quitté la région parisienne, ou qui n'ayant pas encore d'établissement en province ont choisi d'y localiser leur expansion, nous a paru susceptible d'enrichir la connaissance géographique des traductions provinciales de la décentralisation et d'en mieux percevoir l'extrème diversité. I imitant volontairement ici notre étude à cet aspect des choses, étant bien entendu que toutes questions concernant l'espace de départ et les motivations profondes de cette mobilité pourront etre posées ailleurs, nous livrons à propos des manifestations provinciales de la décentralisation quelques réflexions illustrées d'exemples, qui n'ont pour but que d'amorcer une réflexion plus large.

\section{A. Complexité géographique des opérations de transfert}

Nous attachant aux formes prises par ces décentralisations au cours de ces huit années, notre attention est retenue tout d'abord par la complexité des opérations de transfert. Économistes et spécialistes de la gestion ont souvent décrit ces dernières comme des seuils très délicats dans la vie d'une entreprise ; elles apparaissent également très complexes tant dans leur durée que dans leur impact géographique.

Il existe en effet des transferts industriels très linéaires, qui reposent sur une étroite correspondance entre une localisation de départ, une locali- 
sation d'arrivée, et la reconstitution en province, parfois de l'ensemble de l'entreprise, plus souvent d'une unité de production. Ainsi, en 1970, les Établissements A... abandonnent à Paris dans le XIe arrondissement les locaux occupés par leur siège social et leurs fabrications d'outils tranchants, et ils transfèrent l'ensemble à Châtellerault. Dans ce cas, le transfert linéaire n'a fait l'objet que d'une seule opération; il peut souvent se décomposer en plusieurs phases successives. La complexité est alors inhérente à la durée des différentes opérations, lesquelles peuvent s'étaler sur plusieurs années : la Société B... transfère partiellement en 1963 ses fabrications d'Aubergenville à Abbeville, et dans un premier temps spécialise chacun des établissements dans des activités complémentaires. Le regroupement de l'ensemble des fabrications à Abbeville n'est opéré que quatre ans plus tard.

Pourtant, souvent, le transfert est un ensemble ou une suite d'opérations beaucoup plus complexes sur le plan géographique. A l'action de décentralisation, se combinent en effet, fréquemment, restructuration interne de l'entreprise, extension quantitative ou qualitative des fabrications et aussi parfois concentration technique et financière. Le transfert géographique des activités industrielles hors de la région parisienne n'est alors qu'une composante qui prend place dans le remodelage d'ensemble d'un très grand nombre de secteurs industriels en France. Dans ces conditions, la correspondance très stricte entre une localisation de départ et une localisation d'arrivée n'est plus du tout systématique; nous avons relevé ici quelques modalités de transfert caractéristiques par leur fréquence.

Quand il s'insère dans une restructuration générale de l'entreprise, le transfert peut se faire par étapes géographiques. Nous entendons par là que toute émigration d'activités industrielles d'un point initial en région parisienne n'est pas nécessairement perceptible sous sa forme originelle à son arrivée au lieu d'implantation, choisi en province pour accueillir de nouvelles activités. Le transfert s'est effectué par étapes géographiques qui en ont progressivement modifié le contenu. Le programme réalisé par la Société C... en 1967 peut en être une illustration : elle crée dans la Vienne un établissement industriel destiné à assurer l'ensemble de la branche chaussures de l'entreprise; elle abandonne l'usine de Puteaux qui fabriquait jusque-là des accessoires pour l'industrie automobile et transfère ces fabrications à Montargis où elle dispose des locaux libérés par le déménagement des installations du département chaussures dans la Vienne.

Traduisant la volonté de l'entreprise d'opérer une concentration technique, le transfert se fait convergent : à plusieurs localisations de départ ne correspond plus alors qu'un point d'arrivée. Dans ce cas, la suppression d'établissements peut ne concerner que la région parisienne, ou entraîner parallèlement la disparition d'établissements provinciaux. Pour concrétiser ces remarques, et seulement à titre d'exemple, observons ici le déroulement des transferts opérés par la Société D... qui avait avant 1962 à Paris son siège social et deux établissements, l'un dans le XIe arrondissement, l'autre dans le $\mathrm{XX}^{\mathrm{e}}$. Elle transfère à Amiens à cette date les fabrications de son établis- 
sement du XXe, lequel disparaît; en 1965, elle y regroupe l'ensemble de ses activités, abandonnant ses installations du XIe arrondissement. Cette mème société qui n'a plus en région parisienne aucun moyen de production absorbe, en 1969, la Société X..., supprime son seul établissement parisien et en déménage toutes les installations à Amiens dans son unique usine. Nous remarquons que cette Société procède en sept ans à trois opérations de transfert, lesquelles correspondent à la suppression en région parisienne de trois établissements et convergent vers une même unité de production provinciale.

Dans ce même contexte économique de réorganisation générale de l'industrie française, d'une localisation en région parisienne il peut $\mathrm{y}$ avoir transfert divergent vers deux ou plusieurs centres provinciaux, appelés à accueillir respectivement leur part d'activités jusque-là réunies en un même lieu. Tel apparaît le transfert effectué en 1969 par la Société E... d'Issy-lesMoulineaux, où elle libère d'importantes surfaces, vers le Bas-Rhin et la Vienne qui accueillent ces nouvelles fabrications.

Parfois, enfin, une implantation industrielle dite décentralisée ne correspond à aucun transfert apparent d'activité, soit qu'aucune surface industrielle n'ait été libérée en région parisienne, soit même qu'il y ait eu à celte occasion extension de celle-ci. Selon les entreprises, cette absence de transfert découle de réalités très diverses, qui infléchissent sans aucun doute la signification géographique de la décentralisation. Deux situations nous semblent représentatives de cette disparité : dans l'une, l'extension ou la diversification des moyens de production se fait au seul bénéfice d'une implantation provinciale; dans l'autre, il nous parait possible de parler de transfert invisible de fabrications. Dans ce dernier cas, en effet, l'extension en province correspond pour l'entreprise à la volonté de supprimer le recours aux façonniers ou aux sous-traitants sis en région parisienne et de ne plus faire appel à des services extérieurs pour ces travaux. C'est dans le cadre d'une politique interne semblable que par exemple la Société F... étend son établissement de Châtellerault pour y transférer des fabrications jusque-là confiées à des sous-traitants parisiens. Il y a bien transfert invisible d'activités, au détriment de ceux-là mêmes qui en détenaient la charge en région parisienne. On assiste en quelque sorte alors à une forme de confiscation d'activité au bénéfice d'une localisation provinciale.

Au terme de ces réflexions, il nous faut noter que la complexité des formes de transfert va généralement croissant avec la taille des entreprises opérantes et leur assise géographique.

Si nous avons attaché une telle importance à leur analyse, c'est qu'elle nous paraît apporter quelque éclairage à la notion même de décentralisation qui s'incarnant dans les faits s'est peut-être éloignée des schémas ou trop théoriques ou trop vagues qui en avaient été donnés. Cette migration des activités industrielles de la région parisienne vers la province s'insère d'une part dans l'évolution générale des structures financières et techniques de l'industrie française à laquelle nous assistons aujourd'hui, et d'autre part 
dans la durée mème du processus de décentralisation, qui fait que s'éloignent les temps où industries et milieux économiques parisiens et provinciaux s'ignoraient presque totalement. Par la décentralisation entre autres, depuis vingt ans, des liens se sont tissés, les relations se sont multipliées, et la complexité des opérations de transfert nous paraît en être un indicateur non négligreable. Enfin cette approche géographique des transferts nous a semblé favoriser la compréhension de la diversité et de la dynamique des formes d'implantations industrielles décentralisées.

\section{B. Formes de la traduction en province de la décentralisation}

La manifestation en province de cette immigration retiendra donc ici notre attention.

Celle-ci suscite souvent en effet dans les régions françaises la création de nouveaux établissements industriels. I es exemples abondent; la connaissance en est relativement aisée, par l'analyse des statistiques locales et régionales et par l'enquête; l'annonce de ces créations fait très souvent l'objet, de la part des responsables locaux, d'une large publicité, et nous avons pu noter que les géographes y ont jusqu'ici prêté une particulière attention. Ceci justifie-t-il pourtant que l'on attribue toutes les créations de nouveaux établissements industriels en province à la décentralisation ?' En revanche, l'examen des relations existant entre les différents types de créations ne présenterait-il pas un grand intérêt?

Toutefois cette émigration d'activités industrielles parisiennes peut se traduire différemment en province, la signification et l'importance du point de vue de l'industrialisation régionale n'en étant pas moins profondes, mais la connaissance beaucoup plus délicate pour le géographe. La manifestation en province de nombreuses opérations de transfert est l'extension d'établissements déjà existants qui sont, soit le résultat de créations décentralisées antérieures, soit tributaires dès leur origine d'une localisation provinciale sans relation avec la décentralisation. Supports de cette dernière, ils sont par elle fortifiés ou transformés. Nous croyons pouvoir noter qu'avec, dans certaines régions, l'apparition progressive au cours des dernières années d'un tissu industriel un peu plus dense, cette traduction provinciale de la décentralisation semble avoir pris une place plus grande. Son identification parait donc recouvrir un assez grand intérêt. Nous ne citerons ici que deux exemples, mais ceux-ci pourraient être multipliés et diversifiés à l'extrôme. Revenons sur le cas cité précédemment de la Société E..., productrice de matériel électrique. Le transfert de l'ensemble de ses moyens de production dans la Vienne el dans le Bas-Rhin ne suscite aux points d'arrivée aucune création d'établissements nouveaux mais simplement l'extension de deux unités de fabrications existantes. Quand la Société G..., en 196/, abandonne son usine parisienne du $X X^{e}$ arrondissement, elle regroupe l'ensemble de ses fabrications à Feuquières-en-Vimeu où elle possède un etablissement dont l'origine est fort ancienne. 
Enfin certains transferts n'ont sur le point d'arrivée aucun effet apparent extérieur et immédiat, tandis que l'influence profonde peut en ètre considérable. Il s'agit, le plus souvent, d'entreprises provinciales qui, en difficulté pour des raisons diverses, sont rachetées par des entreprises concurrentes dont le siège social et l'essentiel des fabrications sont localisés en région parisienne. Ces dernières transfèrent en province une part plus ou moins grande de leurs installations; ce faisant, elles maintiennent la nature et le niveau de la production des unités menacées, sans qu'il y ait apparemment solution de discontinuité. I'établissement ainsi fortifié devient alors pour l'entreprise parisienne, soit le plus important centre de production, soit une base d'extension future possible. Une telle opération de survie peut n'être suivie que quelques années plus tard de transformations tangibles pour un observateur extérieur. M. M. Krust (18) a évoqué dès 1961 des situations assez proches à propos des sociétés Cotelle et Foucher, Timwear, Laval. Au cours des dernières années, dans le contexte de concentration de l'industrie française précédemment évoqué, les cas analogues semblent s'être multipliés.

En conclusion des remarques qui précèdent, nous mentionnerons qu'un très grand nombre d'implantations décentralisées ne prennent forme que progressivement et selon des modèles très divers : créations, extensions, maintien en activité, mais aussi créations suivies d'extensions successives, extensions ou créations combinées à la disparition d'autres établissements industriels provinciaux, autant de processus, de formes qui risquent d'avoir des significations géographiques différentes et qu'il nous semble précieux de bien connaître.

\section{Approche qualitative des implantations décentralisées}

L'approche qualitative de ces implantations doit tenir le plus grand compte de cette progressivité. Il est en effet important de cerner la nature de l'établissement industriel initialement implanté en province (effet direct ou pas de la décentralisation) par sa taille, ses activités, sa place dans l'entreprise, et le partage qualitatif Paris-province sur lequel il repose; mais ce qu'il faut tout aussi bien essayer de connaître, c'est, sous l'effet des opérations ultérieures de transfert, l'évolution qualitative de cet établissement et le nouvel équilibre qui se crée au sein de l'entreprise, entre la ou les localisations parisiennes et la ou les localisations provinciales. Il peut certes se faire, nous l'avons dit, que l'unité industrielle provinciale regroupe dès sa création ou a la suite d'un seul apport toutes les fabrications et tous les services de l'entreprise ; mais il s'agit le plus souvent de petites entreprises et de cas statistiquement peu nombreux qui, quoique non négligeables, ne retiendront pas ici notre attention. Quand l'unité provinciale se constitue progressivement, elle peut se voir attribuer initialement des orientations très spécifiques, reposant sur différentes conceptions du partage des attributions Paris-province. ( $u a t$ re situations nous paraissent significatives par leur fréquence. Dans un 
premier cas l'ensemble des'fabrications est confié à l'implantation provinciale. Mais certaines entreprises Tn'assignent à l'établissement décentralisé qu'une partie de leurs fabrications. L'affectation est alors parfois tributaire d'une spécialisation verticalędes unités de production; ainsi la Société C... confère à son établissement de Châtellerault la responsabilité de l'ensemble des fabrications de son département de production de chaussures. Ailleurs, le partage est dicté parlles types de fabrication : à l'usine maintenue en région parisienne, à proximité des bureaux d'études et des laboratoires de recherche, est confiée la fabrication de prototypes, tandis que l'établissement décentralisé est spécialisé dans les fabrications de grande série. Enfin certaines entreprises opèrent une dissociation géographique des différentes étapes de la fabrication d'un même produit. Ces attributions diverses donnent aux implantations décentralisées des orientations particulières et leur créent des réseaux de relations originaux dont il conviendrait de bien connaître les flux alors que ces organismes, étrangers pour tout ou partie, s'insèrent dans un espace industriel déterminé.

Mais il s'avère, si l'on s'intéresse à la dynamique de cette forme d'industrialisation régionale, que les choix initiaux sont loin d'être toujours irrévocables, une entreprise étant un organisme vivant. Quand plusieurs opérations de transfert se succèdent, il y a presque toujours élargissement et enrichissement de la base de cette dernière : élargissement quantitatif, mais aussi élargissement qualitatif. Nous entendons par cette dernière expression que l'établissement provincial peut voir se diversifier ses activités tant au niveau des fabrications, par l'élargissement de ses attributions, qu'à celui des aptitudes à la préparation, à la conception ou même à la gestion. On observe alors, à l'avantage de l'établissement provincial, le remodelage de certains choix; telle est par exemple l'évolution à Châtellerault de l'établissement de la Société H..., créé en 1964 pour se voir confier les fabrications de grande série. En 1969, les bureaux d'études et les fabrications de prototypes quittent la région parisienne et lui sont rattachés, ce qui a pour conséquence immédiate la création d'une centaine d'emplois dont la répartition qualitative transforme largement la structure antérieure. L'observation faite à ce niveau ne cerne qu'un des aspects du remodelage, l'appréhension de cette évolution par le seul biais des emplois ne nous paraît pas totalement satisfaisante. L'établissement finalement constitué peut largement différer du noyau initial, tant par la place qu'il occupe dans l'entreprise et l'équilibre qu'il y maintient que par les liens qu'il a établis avec l'espace d'accueil. L'analyse géographique nous semble devoir tirer parti de la constatation de telles évolutions.

\section{Dynamisme des établissements façonnés par la décentralisation}

Quels que soient les processus de constitution de ces unités industrielles, l'analyse ne saurait se limiter à l'élape de la fin des transferts. Ignorée des statisticiens, peu abordée par les géographes qui se sont surtout intéressés 
¿ la décentralisation dans sa première décennie, l'évolution de ces établissements provinciaux dans les années qui suivent la fin des transferts n'a que peu retenu l'attention jusqu'ici. Cette évolution est certes tributaire de nombreux facteurs dont quelques-uns échappent pour tout ou partie au fait de la décentralisation. Certains d'entre eux sont solidaires de la taille de l'entreprise, de sa politique interne, de ses relations et de ses liens extérieurs; d'autres dépendent de la branche d'activité el des conditions générales du marché. Pourtant, si le géographe s'attache à cet aspect des choses, il doit bien constaler que certains de ces établissements, voire de ces entreprises, connaissent dans la phase de leur existence postérieure aux transferts une forte expansion, allant même jusqu'à l'éclatement en de nouveaux établissements sur place ou dans la région d'accueil, tandis que d'autres survivent mal et disparaissent. Partant de ce constat, il nous paraît important d'essayer de cerner dans ces croissances, ces stagnations ou ces déclins, la part relative des facteurs exogènes au milieu d'accueil, et aux organismes décentralisés eux-mèmes, afin de mieux appréhender dans ces évolutions ultérieures celle qui revient aux milieux géographiques d'implantation et à la nature des relations qui ont pu se créer entre ces derniers et les nouvelles industries décentralisées. Ne pourrait-on alors tenter d'analyser pour différents espaces géographiques le dynamisme propre de ces industries importées et donc quelques-uns des effets géographiques fondamentaux d'une telle forme d'industrialisation ?

Ainsi nous sont apparues les traductions provinciales de la décentralisation industrielle au cours des huit dernières années. Issues d'un mouvement migratoire très spécifique, elles ont retenu notre attention par leur diversité et la complexité de leur évolution. Elles s'avèrent également solidaires des mouvements de restructuration interne de l'industrie française et du tissu industriel dans lequel elles s'insèrent. Les quelques remarques qui ont précédé n'avaient pour but que de tenter d'en dégager quelques-uns des aspects originaux.

SIGNIFICATION GÉOGRAPHIQUE DES IMPLANTATIONS INDUSTRIELLES DÉCENTRALISEES EN PROVINCE. - Résumé. - La politique de décentralisation industrielle semble avoir joué depuis une vingtaine d'années en France un rôle important dans la mobilité géographique de certaines activités industrielles. Les pouvoirs publics ont encouragé un processus, entrevu autant comme une émigration des activités industrielles localisées en région parisienne que comme une orientation hors de cette dernière des effets de leur croissance. Ces acceptions sont d'autant plus vagues que l'on s'attache à l'étude de la projection spatiale de ce mouvement. Les géographes français, très tôt intéressés par ce dernier, se sont jusqu'ici peu préoccupés de l'analyse des formes d'implantations et de leur dynamisme de croissance. Il nous est apparu nécessaire de présenter les aspects les plus caractéristiques de la complexité géographique des opérations de transferts industriels observés afin de mieux dégager les formes originales des implantations décentralisées en province, lesquelles doivent être appréhendées dans la dynamigue même de leur évolution. 
TIIE GEOGRAPIIC SIGNIFICANCE OF THE IETENTRALIZED INDUSTRIAL SETTLEMENTS IN THE PROVINCES. - Abstract. - The policy of industrial decentralization seems to have played an important role in the geographical mobility of some industrial activities in France for twenty years or so. The administration supports a system which is considered as a move of industrial activities located in the l'aris region, as much as an orientation far from this region of the effects of their growth. Those definitions are so much vague as the study of the spatial projection of this move is looked upon. The French geographers were very soon interested in that move and did not worry very much about the analysis of the settlements forms and of their growth dynamism. It appeared to us as a necessity to present the most characteristic aspects of the geographical complexity the industrial moves show in order to determine better the original types of decentralized settlements which must be studied through the very dynamic of their evolution.

\section{Bibliographie}

AtLAS

(1) Atlas de Normandie, Caen, 1966 : carte E8, a les créations industrielles „, commentaire par F. J. Gay.

(2) Atlas de Paris et de la région parisienne, Paris, Berger-Levrault, 1967 : « Évolution industrielle décentralisation (1954-1964) n, carte 73-2 et commentaire par J. Bastié et $P$. Trolliet.

Ouvrages

(3) Babonaux Y., Villes et régions de la Loire moyenne, Touraine, Blésois, Orléanais, Paris, Sabri, 1966, $744 \mathrm{p}$.

(4) Dessus G., Grorge P., Weurlesse A., Matériaux pour une géographie volontaire de l'industrie française, Paris, $1949,149 \mathrm{p}$.

(5) George P., La France, Paris, P.U.F., $2^{\text {eme èd. } 1970 .}$

(6) LA BASSe J., L'Organisation de l'espace, Paris, Hermann, 1965.

(7) Pinchemel P., La France, 2 vol., Paris, A. Colin, 2e éd. 1969.

(8) Rochefort M., Bidault C., Petit M., Aménager le territoire, Paris, Le Seuil, 1970.

(9) Institut d'Études politiques de Grenoble : Aménagemen du Territoire et développement régional, Grenoble, 5 vol. : 1968, 1969, 1970, 1971, 1972.

Articles, Notes et Publications diverses

(10) BAвoNauX Y., "La décentralisation devant le problème de la main-d'œuvre du Loir-et-Cher", Norois, 1959, p. 47-52.

(11) Bastí́ J., "Paris ville industrielle ", Notes et Études documentaires, no 3690-3691, 1970.

(12) Burgel G., "La main-d'ouvre des établissements décentralisés : l'exemple de l'Eure ", Annales de Géographie, no 404, 1965, p. 416-431.

(13) Chesnais M., a Bilan de la décentralisation industrielle en Eure-et-Loir ", Norois, 1964, p. 521-525.

(14) Frémont A., Ambois M., Chesnais M., "Argentan, une petite ville de Basse-Normandie, ranimée par la décentralisation industrielle $n$, Norois, 1964, p. 419-437.

(15) Geonge P., " Nécessités et difficultés d'une décentralisation industrielle en France ", Annales de Géographie, no 377, 1961, p. 25-36.

(16) Gerbautt J., "Le développement industriel de l'agglomération dijonnaise n, Annales de Géographie, no 441,1971, p. 534-553.

(17) Gounier J., "Notes sur la décentralisation industrielle entre Seine et Loire ", Annales de Géographie, no 402, 1965, p. 220-223.

(18) KRUST M. M., "Une forme de décentralisation industrielle : la réanimation des grandes villes situées entre 100 et 200 kilomètres de Paris. Le cas de Reims ", Bulletin du Comité des Travaux historiques et scientifiques: Section de géographie, tome LXXIV, 1961, p. 55-72.

(19) Meynier A. et Le Guen M., "Rennes. Les grandes villes françaises ", Notes et Etudes documentaires, no $3267,1966$.

(20) Parry C., a Un exemple de décentralisation industrielle : la dispersion des usines de la Radiotechnique à l'ouest de l'aris ^, Annales de G'égraphie, no 390, 1963, p. 148-161. 
(21) Palienne J.-L. et Riquet P., "La décentralisation industrielle et le Bassin Parisien", Cahiers de l'I.A.U.R.P., fasc. 6, 1966.

(22) Saint-Julien Th., "La Rochelle et la décentralisation industrielle ", Annales de Géographie. no 442,1971, p. 687.706.

Thèses de doctorat de zeme cycle

(23) Bidatit C., La Décentralisation industrielle dans le Maine, Thèse de $3^{\text {eme }}$ cycle, Paris, 1970, $139 \mathrm{p}$.

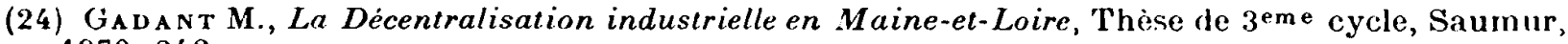
$1970,242 \mathrm{p}$.

(25) Tholift P., La Décentralisation industrielle de la région parisienne, Bilans et aspects, 1950-1964, Thèse de $3^{\text {eme }}$ cycle, Paris, 1969.

(26) Petit M., Expansion industrielle et éolution démographique en Bourgogne, Thèse de 3 eme cycle, Paris, 1971. 